\title{
MOTION OF HYPERSURFACES BY CURVATURE
}

\author{
MAT LANGFORD
}

(Received 16 June 2015; first published online 19 August 2015)

2010 Mathematics subject classification: primary 53C44; secondary 35K55.

Keywords and phrases: curvature flows, convexity estimates, cylindrical estimates, noncollapsing.

We study the behaviour of (nonconvex) solutions of a large class of fully nonlinear curvature flows; specifically, we consider the evolution of smooth, connected, closed, immersed hypersurfaces of Euclidean spaces whose pointwise normal speed is prescribed by a smooth, monotone function of their curvature which is homogeneous of degree one.

It is well known that solutions of such flows necessarily suffer finite time singularities. On the other hand, under various natural conditions, singularities are characterised by a curvature blow-up. Our first main area of study concerns the asymptotic behaviour of the curvature at a singularity. We first prove a quantitative convexity estimate for positive solutions (that is, solutions moving with inward normal speed everywhere positive) under one of the following additional assumptions: either the evolving hypersurfaces are of dimension two, or the flow speed is a convex function of the curvature. Roughly speaking, the convexity estimate states that, for positive solutions, the normalised Weingarten curvature operator is asymptotically nonnegative at a singularity $[4,5]$. We then prove a family of cylindrical estimates for flows by convex speed functions. Roughly speaking, these estimates state that, for $(m+1)$ positive solutions (that is, solutions with $(m+1)$-positive Weingarten curvature), the Weingarten curvature is asymptotically $m$-cylindrical at a singularity unless it becomes $m$-positive [1]. The convexity and cylindrical estimates yield a detailed description of the possible singularities which may form under surface flows and flows by convex speeds. Moreover, they are uniform across the class of solutions with given dimension and flow speed, and given initial volume, diameter and curvature hull, which should make them useful for applications such as the development of flows with surgeries.

Our second main area of study concerns the development, in the fully nonlinear setting, of the recently discovered noncollapsing phenomena for the mean curvature

Thesis submitted to the Australian National University in August 2014; degree approved on 20 February 2015; supervisors Ben Andrews and James McCoy.

(c) 2015 Australian Mathematical Publishing Association Inc. 0004-9727/2015 \$16.00 
flow; namely, we prove that embedded solutions of flows by concave speeds are interior noncollapsing, whilst embedded solutions of flows by convex or inverse-concave speeds are exterior noncollapsing $[2,3]$. The noncollapsing results complement the above curvature estimates by ruling out certain types of asymptotic behaviour which the curvature estimates do not. (This is mainly due to the nonlocal nature of the noncollapsing estimates.) As a particular application, we show how noncollapsing gives rise to a particularly efficient proof of the Andrews-Huisken theorem on the convergence of convex hypersurfaces to round points under such flows.

\section{References}

[1] B. Andrews and M. Langford, 'Cylindrical estimates for hypersurfaces moving by convex curvature functions', Anal. PDE 7 (2014), 1091-1107.

[2] B. Andrews and M. Langford, 'Two-sided non-collapsing curvature flows', Ann. Sc. Norm. Super. Pisa Cl. Sci., to appear.

[3] B. Andrews, M. Langford and J. McCoy, 'Non-collapsing in fully non-linear curvature flows', Ann. Inst. H. Poincaré Anal. Non Linéaire 30 (2013), 23-32.

[4] B. Andrews, M. Langford and J. McCoy, 'Convexity estimates for hypersurfaces moving by convex curvature functions', Anal. PDE 7 (2014), 407-433.

[5] B. Andrews, M. Langford and J. McCoy, 'Convexity estimates for hypersurfaces moving by curvature functions', J. Differential Geom. 99 (2015), 47-75.

MAT LANGFORD, Fachbereich Mathematik und Informatik, Freie Universität Berlin, Arnimallee 3, 14195 Berlin, Germany

e-mail: mathew.langford@fu-berlin.de 